\title{
Grado de deterioro cognitivo de los adultos mayores institucionalizados en dos hogares para ancianos del área metropolitana de Bucaramanga - Santander, Colombia
}

\author{
Degree of cognitive impairment of older adults institutionalized in two homes for the elderly in the metropolitan \\ area of Bucaramanga-Santander, Colombia
}

Katherine del Consuelo Camargo-Hernández ${ }^{*}$ orcid.org/0000-0003-4840-3098

Elveny Laguado-Jaimes' orcid.org/0000-0001-7169-6912

1 Facultad de Enfermería, Universidad Cooperativa de Colombia. Bucaramanga, Colombia.

Fecha de recepción: Octubre 28 - 2016

Fecha de revisión: Marzo 1 - 2016

Fecha de aceptación: Agosto 11 - 2017

Camargo-Hernández $K$ del C, Laguado-Jaimes E. Grado de deterioro cognitivo de los adultos mayores institucionalizados en dos hogares para ancianos del área metropolitana de Bucaramanga - Santander, Colombia. Univ. Salud. 2017;19(2):163-170. DOI: http://dx.doi.org/10.22267/rus.171902.79

\begin{abstract}
Resumen
Introducción: El deterioro cognitivo es uno de los problemas que se presentan con mayor frecuencia en la población adulta mayor como consecuencia del envejecimiento. Objetivo: Determinar el grado de deterioro cognitivo de los adultos mayores institucionalizados en dos hogares para ancianos del área metropolitana de Bucaramanga, Santander, Colombia. Materiales y métodos: Estudio cuantitativo con diseño descriptivo, de corte transversal, con una población de 165 y una muestra de 60 adultos mayores de dos hogares para ancianos, cuyos criterios de inclusión fueron: edad entre 65 y 95 y más años, presencia o ausencia de patología crónica. Se utilizó el muestreo no probabilístico por conveniencia, y se aplicó el instrumento: MMSE (Examen Cognoscitivo Mini-Mental). Resultados: La población adulta mayor que participó en el estudio oscila entre las edades de 75 a 94 años, existió predominio del género masculino; la mayoría se encontraban en nivel básico primario, tenían contacto familiar, el tiempo de institucionalización fue de 1 a 5 años. Conclusiones: El 41,7\% de los adultos mayores estudiados presentaron deterioro cognitivo grave y requieren intervenciones de enfermería enfocadas en la terapia cognitiva.
\end{abstract}

Palabras clave: Adulto mayor; hogares para ancianos; institucionalización; deterioro cognitivo leve; envejecimiento. (Fuente: DeCS, Bireme).

\begin{abstract}
Introduction: Cognitive impairment is one of the most frequent occurring problems in the older adult population as a result of ageing. Objective: Determine the degree of cognitive impairment of older adults institutionalized in two homes for the elderly in the metropolitan area of Bucaramanga, Santander in Colombia. Materials and methods: A quantitative study was made by using a cross-sectional descriptive design with a population of 165 and a sample of 60 older adults from two homes for the elderly, whose inclusion criteria were: age between 65 to 95 or more and presence or absence of chronic pathology. A non-probabilistic sampling was used for convenience, and the instrument MMSE (Mini-mental state examination) was applied. Results: The older adult population who participated in the study is between the ages of 75 to 94 . Most of them were male, had a primary school level, had family contact and the time for institutionalization was 1 to 5 years. Conclusions: $41.7 \%$ of older adults studied showed severe cognitive impairment and require nursing interventions focused on cognitive therapy.
\end{abstract}

Keywords: Aged; homes for the aged; institutionalization; mild cognitive impairment; aging. (Source: DeCS, Bireme). 


\section{Introducción}

El ser humano pasa por una serie de etapas en todo el trayecto de su vida, la última de ellas es la vejez, la cual es considerada por Arango y Ruiz, como "el último escalón del ciclo vital"(1). Actualmente se considera que los adultos mayores, son personas con edades superiores a los 65 años; según la Organización Mundial de la salud (OMS), la población adulta mayor ha tenido un incremento rápido y significativo en todo el mundo, se estimó que entre los años 2015 y 2050, los adultos mayores podrían pasar de un 12 a un $22 \%$, es decir casi el doble de la población actual(2).

En Colombia, la población adulta mayor va aumentando rápidamente, según las proyecciones del Departamento Administrativo Nacional de Estadística (DANE), para el año 2014, del total de 47.661.787 habitantes, 5.146 .251 , pertenecían a población mayor de 60 años y para el año 2020, esta población incrementará a 6.440 .778 de un total de 50.911.747 habitantes(3).

El envejecimiento es considerado como un proceso en donde ocurre un deterioro progresivo en el organismo, que conlleva a alteraciones morfológicas, funcionales, psicológicas y bioquímicas provocando vulnerabilidad en situaciones de estrés y culmina con la muerte(4). Dentro de las alteraciones más destacadas en la etapa de la vejez, se encuentra el deterioro o trastorno cognitivo, este puede ocurrir por "disminución de habilidades mentales asociadas al envejecimiento, estados depresivos, deterioro cognitivo leve y demencia"(5).

El deterioro cognitivo es uno de los problemas que se presentan con mayor frecuencia en la población adulta mayor como consecuencia del envejecimiento. La función cognitiva es el funcionamiento integral de la percepción, atención, habilidad, orientación, memoria, lenguaje y cálculo, los cuales en el envejecimiento sufren algún grado de deterioro. Entre algunas de las funciones de la memoria que no se afectan notablemente o permanecen estables son la memoria semántica y la de procedimiento. En la misma medida las funciones de la memoria que más se afectan en el adulto mayor, son la memoria de trabajo, episódica y prospectiva(6).

En esta investigación se determinó el grado de deterioro cognitivo de los adultos mayores institucionalizados en dos hogares para ancianos del área metropolitana de Bucaramanga, Santander, Colombia. Los resultados de esta investigación, pueden contribuir de forma exitosa para la creación de intervenciones en enfermería, de acuerdo con los criterios del lenguaje enfermero estandarizado, para estimular el área cognitiva en este grupo poblacional.

\section{Materiales y métodos}

Estudio cuantitativo, con diseño descriptivo, de corte transversal. La población estuvo conformada por 165 adultos mayores de dos hogares para ancianos del área metropolitana de Bucaramanga, Santander, Colombia. La muestra para esta investigación fue de 60 adultos mayores, los cuales cumplían con los criterios de inclusión, de tener edades oscilantes entre los 64 a 95 o más años de edad y presencia o ausencia de patología crónica.

Se consideró este antecedente debido a la inclusión de la variable uso de medicamentos dentro del instrumento de medición, para la muestra se seleccionaron patologías cardiovasculares, respiratorias, metabólicas, por ser las más frecuentes. Además al realizar la recolección de los datos, se encontraron otras alteraciones en el estado de salud presentes en la población adulta mayor como problemas visuales, auditivos, gastrointestinales y renales. Los criterios de exclusión fueron los siguientes: adultos mayores totalmente dependientes en sus actividades de la vida diaria, con alteraciones sensoriales, con patologías invalidantes, participación activa en un grupo o programa de interacción social o con deterioro intelectual severo. 
Se utilizó muestreo no probabilístico por conveniencia, con la aplicación del instrumento MMSE (Examen Cognoscitivo Mini-Mental) de Lobo et al(7)., el cual fue validado al idioma español por estos autores donde se valoran las variables: orientación temporal, orientación espacial, fijación, atención y cálculo, memoria, nominación, comprensión, lectura, escritura y dibujo; además se tomaron datos sociodemográficos como: edad, género, escolaridad, tiempo de institucionalización, contacto familiar y diagnóstico médico. El tipo de análisis de los resultados fue descriptivo, teniendo en cuenta datos de frecuencias y porcentajes, con utilización del software IBM SPSS versión 22.

\section{Consideraciones éticas}

El estudio de investigación se desarrolló previo concepto del Comité de Ética y la Dirección de Investigaciones de la Universidad Cooperativa de Colombia, seccional Bucaramanga. Esta investigación se clasifica como estudio sin riesgo, de acuerdo a lo establecido en el numeral a del Artículo 11, de la Resolución 8430 del 04 de Octubre de 1993 del Ministerio de Salud de la
República de Colombia(8), se diligenció un consentimiento informado, en donde se explicó el propósito, el procedimiento y la participación voluntaria en el estudio a cada adulto mayor antes de realizarse la recolección de los datos, tal como lo establece el Tribunal Departamental Ético de Enfermería Región Nororiental en el Capítulo II Artículo 6(9).

\section{Resultados}

En cuanto a la variable edad se evidenció que la mayoría de los adultos mayores se encontraron entre edades de 75 a 94 años, es decir un $73 \%$ de la población total, en cuanto al género los hallazgos indican que el 55\% (33) de los adultos mayores pertenecen al género masculino por una diferencia mínima de seis participantes versus género femenino $(27 ; 45 \%)$. Al analizar la variable escolaridad se pudo evidenciar que la población adulto mayor cuenta con nivel de escolaridad primaria (34; 57\%), sin embargo, hay un alto índice de personas analfabetas (15; $25 \%)$, lo cual pudo ser un factor de riesgo para presentarse deterioro cognitivo (Tabla 1).

Tabla 1. Edad, género y escolaridad de la población total

\begin{tabular}{|c|c|c|c|c|c|c|c|}
\hline \multirow[b]{2}{*}{ Género } & \multirow[b]{2}{*}{ Escolaridad } & & \multicolumn{2}{|c|}{ Edad (años) } & \multirow[b]{2}{*}{ 85-94 } & \multirow[b]{2}{*}{95 o más } & \multirow[b]{2}{*}{ Total } \\
\hline & & & $64-74$ & $75-84$ & & & \\
\hline \multirow{10}{*}{ Femenino } & \multirow{2}{*}{ Analfabeta } & $\mathrm{n}$ & 1 & 1 & 3 & 0 & 5 \\
\hline & & $\%$ & 1,67 & 1,67 & 5,00 & 0,00 & 8,34 \\
\hline & \multirow{2}{*}{ Primaria } & $\mathrm{n}$ & 4 & 6 & 4 & 1 & 15 \\
\hline & & $\%$ & 6,67 & 10,0 & 6,67 & 1,67 & 25,01 \\
\hline & \multirow{2}{*}{ Secundaria } & $\mathrm{n}$ & 0 & 1 & 4 & 1 & 6 \\
\hline & & $\%$ & 0 & 1,67 & 6,67 & 1,67 & 10,01 \\
\hline & \multirow{2}{*}{ Superior } & $\mathrm{n}$ & 0 & 0 & 1 & 0 & 1 \\
\hline & & $\%$ & 0,0 & 0,0 & 1,67 & 0,0 & 1,67 \\
\hline & \multirow{2}{*}{ Total } & $\mathrm{n}$ & 5 & 8 & 12 & 2 & 27 \\
\hline & & $\%$ & 8,33 & 13,33 & 20,00 & 3,33 & 44,99 \\
\hline \multirow{8}{*}{ Masculino } & \multirow{2}{*}{ Analfabeta } & $\mathrm{n}$ & 3 & 4 & 3 & 0 & 10 \\
\hline & & $\%$ & 5,00 & 6,67 & 5,00 & 0 & 16,67 \\
\hline & \multirow{2}{*}{ Primaria } & $\mathrm{n}$ & 7 & 10 & 2 & 0 & 19 \\
\hline & & $\%$ & 11,67 & 16,67 & 3,33 & 0 & 31,67 \\
\hline & \multirow{2}{*}{ Secundaria } & $\mathrm{n}$ & 1 & 2 & 1 & 0 & 4 \\
\hline & & $\%$ & 1,67 & 3,33 & 1,67 & 0 & 6,67 \\
\hline & \multirow{2}{*}{ Total } & $\mathrm{n}$ & 11 & 16 & 6 & 0 & 33 \\
\hline & & $\%$ & 18,33 & 26,66 & 10,00 & 0 & 55,01 \\
\hline
\end{tabular}

Se encontró que la mayoría de los adultos mayores $(45 ; 75 \%)$ tienen contacto con algunos de sus familiares y el tiempo que llevan de estar institucionalizados es de 1-5 años (33; 55\%) (Tabla 2). 
Tabla 2. Contacto familiar y tiempo de institucionalización población total

\begin{tabular}{|c|c|c|c|c|c|c|}
\hline & \multicolumn{4}{|c|}{ Tiempo institucionalización } & \multirow[b]{2}{*}{ Total } \\
\hline & & Menor de un año & 1 a 5 años & 6 a 10 años & $\begin{array}{c}16 \text { a } 20 \\
\text { años }\end{array}$ & \\
\hline \multirow{3}{*}{ SI } & $\mathrm{n}$ & 12 & 24 & 6 & 3 & 45 \\
\hline & $\%$ Contacto familiar & 26,7 & 53,3 & 13,3 & 6,7 & 100 \\
\hline & \% Tiempo institucionalización & 85,7 & 72,7 & 66,7 & 75 & 75 \\
\hline \multirow{3}{*}{ NO } & $\mathrm{n}$ & 2 & 9 & 3 & 1 & 15 \\
\hline & $\%$ Contacto familiar & 13,3 & 60 & 20 & 6,7 & 100 \\
\hline & \% Tiempo institucionalización & 14,3 & 27,3 & 33,3 & 25 & 25 \\
\hline
\end{tabular}

De acuerdo a los hallazgos encontrados en los datos socio-demográficos, con respecto a la variable diagnóstico médico (Tabla 3), se puede evidenciar que la patología que se presentó con mayor frecuencia en la población objeto fueron las cardiovasculares $(34 ; 57 \%)$, resaltando que ésta se encuentra acompañada de varias patologías diagnosticadas médicamente en el adulto mayor; en segundo lugar se encuentran las patologías clasificadas como otras, las cuales son entendidas como: problemas visuales, auditivos, renales y gastrointestinales $(15 ; 25 \%)$.

Tabla 3. Diagnóstico médico

\begin{tabular}{lcc}
\hline \multicolumn{1}{c}{ Característica } & \multicolumn{2}{c}{ Consolidado hogar } \\
para ancianos (60)
\end{tabular}

Tabla 4. Mini Examen Cognoscitivo-MEC población total

\begin{tabular}{|c|c|c|}
\hline \multirow{2}{*}{$\begin{array}{c}\text { Característica } \\
\text { Mini Examen Cognsoscitivo-MEC }\end{array}$} & \multicolumn{2}{|c|}{$\begin{array}{c}\text { Consolidado hogar para } \\
\text { ancianos }(60)\end{array}$} \\
\hline & $\mathbf{n}$ & $\%$ \\
\hline Probablemente sin deterioro & 20 & 33,3 \\
\hline Probablemente con deterioro & 1 & 1,7 \\
\hline Moderada & 14 & 23,3 \\
\hline Grave & 25 & 41,7 \\
\hline Total & 60 & 100 \\
\hline
\end{tabular}

En relación a los resultados, se puede observar que el $33,3 \%$ (20) de adultos mayores intervenidos, se encuentran sin deterioro cognitivo y el $41,7 \%$ (25) se encuentran con deterioro cognitivo grave (Tabla 4).

\section{Discusión}

Los adultos mayores son considerados como una población vulnerable en la sociedad, en esta etapa se presentan alteraciones en las funciones mentales, lo cual conlleva a un deterioro cognitivo en esta población. El deterioro cognitivo, es considerado un trastorno de la salud mental, este trastorno es considerado en la Ley 1616 del 21 de enero de 2013, de Colombia como: Una alteración de los procesos cognitivos $\mathrm{y}$ afectivos del desenvolvimiento considerado como normal con respecto al grupo social de referencia del cual proviene el individuo. Esta alteración se manifiesta en trastornos del razonamiento, del comportamiento, de la facultad de reconocer la realidad y de adaptarse a las condiciones de la vida(10).

En esta investigación se determinó que el $41,7 \%(25)$ de los adultos mayores participantes del estudio tienen deterioro cognitivo grave, seguido de probablemente sin deterioro33,3\% (20) y un $23,3 \%$ (14) con deterioro; mientras que en el estudio de Leite et al(11), de un total de 341 adultos mayores el $60,3 \%$ se encuentran sin deterioro cognitivo y un $33,2 \%$ con deterioro cognitivo. Estos resultados coinciden con los reportados por Díaz et al; en donde se evidenció que el 23\% de los adultos mayores de 65 años prevalecía la demencia como enfermedad neurológica(5), lo cual es un signo de alarma en el área de salud pública y que refleja la importancia que tiene el direccionar los cuidados de 
enfermería con intervenciones como entrenamiento de la memoria, estimulación cognitiva, facilitar el aprendizaje, potencialización de la disposición de aprendizaje, restructuración cognitiva, terapia de reminiscencia y orientación a la realidad en el adulto mayor, para prevenir y mitigar alteraciones del área cognitiva en este grupo poblacional(12).

Estos hallazgos se pueden ver asociados al grado de escolaridad de los participantes del estudio, según los resultados obtenidos en esta variable el $56,7 \%$ de los adultos mayores tenían estudio de primaria y un $25 \%$ era analfabeta. Lo anterior puede convertirse en un factor de riesgo para el desarrollo del deterioro cognitivo de la población adulta mayor, estos datos coinciden con lo enunciado por Rodríguez y Sánchez, en donde hacen relación a la reserva cognitiva como aquel "mecanismo que vincula los niveles educativos bajos con un mayor riesgo de padecer un proceso neurodegenerativo"(13). Además, en el estudio realizado por Abarca et al, se encontró que los participantes con menor puntuación en la valoración del área cognitiva fueron la población analfabeta, en adición a esto, los autores resaltaron la importancia de la educación en el ser humano, puesto que el alto nivel educativo, puede convertirse en un factor protector contra el deterioro cognitivo, presente en la población adulta mayor(14). Lo cual puede direccionar el cuidado de enfermería hacia el nivel Conductual, que hace referencia a los cuidados que respaldan la función psicosocial y pueden facilitar los cambios o modificaciones de los estilos de vida en las personas sujetas de cuidado(12). Por esta razón la Clasificación de Intervenciones de Enfermería (NIC), que permite direccionar los cuidados enfermeros, de acuerdo a las necesidades propias de los sujetos de cuidado.

En esta investigación la edad de los participantes oscila entre los 75 a 94 años 70\% (42) (Tabla 1), estos datos coinciden con lo presentado en la proyección poblacional 2014, realizada por el DANE de Colombia, en donde se estableció que 1.344.409 de adultos mayores se encontrarían en este rango de edades para una población de 47.661.787 habitantes(3); cifra que corrobora el aumento de la población adulta mayor en todo el mundo, e implica un cuidado especializado por parte del profesional de enfermería, el cual fue estipulado en la Ley 911 de Octubre 5 de 2004 por el Tribunal Nacional Ético de Enfermería Región Nororiental, en el capítulo II, Artículo 3, en donde se enuncia que el cuidado de enfermería: Requiere de un juicio de valor y un proceso dinámico y participativo para identificar y dar prioridad a las necesidades y decidir el plan de cuidado de enfermería, con el propósito de promover la vida, prevenir la enfermedad, intervenir en el tratamiento, en la rehabilitación y dar cuidado paliativo con el fin de desarrollar, en lo posible, las potencialidades individuales y colectivas $^{(9)}$.

Además, la edad es considerada otro factor de riesgo para el desarrollo del deterioro cognitivo en la población adulta mayor y los hallazgos de esta investigación, también coincidieron con los reportados por Durán-Badillo et al, en donde se "encontró mayor prevalencia de deterioro en el rango de edad de 81 a 90 años (55,5\%)”(15).

El género es una variable que se debe analizar detalladamente y aunque este estudio reporta que el 55\% de los adultos mayores, pertenecen al género masculino (Tabla 2), por una diferencia mínima de seis participantes, es necesario hacer hincapié que en la población colombiana, según las proyecciones 2014, dadas por el DANE para una población de 47.661.787 habitantes, 23.531.670 son hombres y 24.130.117 son mujeres(3), es decir hay más mujeres que hombres en la población, esto puede ser porque "en la medida que ha aumentado la longevidad, principalmente en el sexo femenino, el índice de masculinidad disminuye"(1). Lo anterior coincide con un estudio realizado por Mías et al, en donde el $78 \%$ de la muestra fueron adultos mayores de género femenino; esta variable fue considerada como un factor de riesgo significativo, en donde la "correspondencia del género femenino, la edad a partir de los 65 años, el bajo nivel de instrucción y del número de hijos parecen ser las variables que más influyen en el estado cognitivo de deterioro en relación a la normalidad"(16) que conlleva a identificar los factores de riesgo para el deterioro cognitivo en la población adulta 
mayor según el género y así, poder garantizar cuidados de enfermería bajo estándares de calidad(9).

En cuanto al contacto familiar, el estudio arrojó que el $75 \%$ (45) de los adultos mayores intervenidos tenían contacto con sus familias; según Ávila, la familia es una red de apoyo en todos los seres humanos, en procesos de enfermedad, salud y muerte(17), en esta investigación este hallazgo puede considerarse como un factor protector en la etapa de la vejez y de acuerdo a lo estipulado en el Tribunal Departamental Ético de Enfermería Región Nororiental, en el capítulo II, Artículo 3, el cuidado de enfermería surge "a partir de la comunicación y relación interpersonal humanizada entre el profesional de enfermería y el ser humano, sujeto de cuidado, la familia o grupo social, en las distintas etapas de la vida, situación de salud y del entorno"(9). Por tanto, es indispensable involucrar las personas cercanas a los adultos mayores, para alcanzar los objetivos propuestos en los planes de cuidados instaurados por el profesional en enfermería.

En la Constitución Política de Colombia de 1991, en el artículo 46, se estableció que "el estado, la sociedad y la familia concurrirán para la protección y la asistencia de las personas de la tercera edad y promoverán su integración a la vida activa y comunitaria"(18).

Una de las estrategias establecidas por los gobiernos, son los hogares para ancianos, en donde se deben realizar intervenciones encaminadas a la protección de este grupo poblacional. Sin embargo para Pinillos y Prieto "la institucionalización se identifica como uno de los factores que actúa en la disminución de autonomía de la persona mayor"(19) puesto que esta población, se enfrenta a situaciones nuevas para esa etapa de la vida como son el entorno, compañeros, estilos de vida muy diferentes a los vividos en las etapas anteriores, aunque estos autores también refirieron que en los adultos mayores que se encuentren con limitaciones que impidan el desarrollo de actividades básicas de la vida diaria, o que vivan solas: Se hace necesario garantizar a las personas mayores su mantenimiento al ingreso a un hogar geriátrico, y como respuesta de la salud pública a las necesidades de esta creciente población, precisa que se aumente la efectividad en todas las formas de cuidado e intervención en torno a la salud de los mismos ${ }^{(19) .}$

De igual forma Melguizo-Herrera et al., enunciaron que: "Aunque algunos piensen que los centros de protección social son lugares de soledad y abandono, en realidad son sitios donde se favorece el contacto y las relaciones sociales. Allí los adultos mayores pueden desarrollar nuevas habilidades y destrezas físicas y sociales, y compartir con gente de su misma edad"(20).

Además, el Tribunal Departamental Ético de Enfermería Región Nororiental en el Título III, Capítulo I, Artículo 11 establece lo siguiente: "El profesional de enfermería deberá garantizar cuidados de calidad a quienes reciben sus servicios. Tal garantía no debe entenderse en relación con los resultados de la intervención profesional, dado que el ejercicio de la enfermería implica una obligación de medios, mas no de resultados"(9).

Los adultos mayores son catalogadas como personas vulnerables, debido a todos los cambios que se presentan en la dimensión física, social y psicológica, la cual es notable en aquellos adultos mayores que se encuentran institucionalizados(15), por tanto, es responsabilidad del profesional de enfermería realizar un cuidado individualizado, bajo estándares de eficiencia y calidad, que contribuya en la prevención y disminución de alteraciones cognitivas en este grupo poblacional.

Esto refuerza la necesidad de realizar intervenciones de enfermería relacionadas con el nivel conductual, en donde se encuentran intervenciones de Terapia Cognitiva(12), que contribuyen al fortalecimiento cognitivo, al enlentecimiento en el proceso degenerativo de algunas enfermedades de salud mental presentes en los adultos mayores, y por ende se puede llegar a mejorar la salud física, mental y la calidad de vida de esta población. 
En cuanto al diagnóstico médico de los adultos mayores participantes en el estudio, se pudo evidenciar que las patologías que se presentaron con mayor frecuencia fueron las que se encontraron en la categoría: cardiovasculares, (13; 21,6\%), además, el 16,6\% (10) de los adultos mayores con estas patologías también presentaban otras, entendidas como: problemas visuales, auditivos, renales, gastrointestinales.

Estos hallazgos coinciden con los obtenidos por León-Arcila et al., en donde las principales enfermedades presentes en la población adulta mayor fueron: trastornos cardiovasculares (cardiopatías, hipertensión arterial, accidentes cerebrovasculares, infartos agudos al miocardio) con un $70 \%$, los cuales pueden considerarse como factores de riesgo para el deterioro cognitivo en la población adulta mayor (21). De allí radica la importancia de implementar las políticas de detección temprana, promoción de la salud y prevención de la enfermedad por parte del profesional sanitario, con el objetivo de detectar enfermedades no transmisibles, disminuir los efectos que traen las mismas en la salud de las personas adultas mayores; generar, fortalecer conductas saludables y modificar conductas que no lo son.

\section{Conclusiones}

El deterioro cognitivo es una situación frecuente en la población adulta mayor institucionalizada, tal como lo muestra el presente estudio, donde se pudo establecer que el $41,7 \%$ (25) de los participantes tienen deterioro cognitivo grave, y la distribución por género es similar $(27 ; 45 \%$ son mujeres y 33; $55 \%$ son hombres), cabe destacar que es una población con nivel de escolaridad de primaria $(34 ; 56,7 \%)$ o analfabeta $(15 ; 25 \%)$. Estos resultados invitan a realizar intervenciones integrales, donde el profesional de enfermería cumple un papel importante en la conservación de la capacidad de la memoria de esta población a lo largo del tiempo.

A partir de este estudio surgen problemas que pueden ser resueltas en futuras investigaciones tales como establecer la asociación entre el contacto familiar, nivel de escolaridad, género, patología y el deterioro cognitivo en el adulto mayor institucionalizado.

\section{Fuente de financiación}

Comité para el Desarrollo de la InvestigaciónCONADI Universidad Cooperativa de Colombia.

\section{Agradecimientos}

Los autores agradecen a los adultos mayores y directivas de los hogares para ancianos del área metropolitana de Bucaramanga, Santander, Colombia que participaron en el proyecto $y$ auxiliares de investigación del proyecto, pertenecientes a la Facultad de Enfermería de la Universidad Cooperativa de Colombia, sede Bucaramanga durante el primer y segundo semestre de 2015.

\section{Conflicto de intereses}

Dentro de esta investigación no hubo conflictos de intereses, no se usaron incentivos o recompensas en el proceso de inducir la investigación.

\section{Referencias}

1. Arango VE, Ruiz IC. Diagnóstico de los adultos mayores de Colombia. Bogotá: Fundación Saldarriaga Concha; 2008. [consultado 2016 Aug 27]; Disponible en: http://www.sdp.gov.co/portal/page/portal/PortalSDP /SeguimientoPoliticas/Politicas\%20Poblacionales/Env ejecimiento\%20y\%20Vejez/Documentacion/A31ACF9 31BA329B4E040080A6C0A5D1C

2. Organización Mundial de la Salud. La salud mental y los adultos mayores [Internet]. Ginebra: OMS; 2016 [consultado 2016 Aug 27]. Disponible en: http://www.who.int/mediacentre/factsheets/fs381/es /

3. Departamento Administrativo Nacional de Estadística. Estimaciones de población 1985-2005 y proyecciones de población 2005-2020 nacional y departamental desagregado por área, sexo y grupos quinquenales de edad [Internet]. Bogotá: DANE; 2015. Disponible en: https://www.dane.gov.co/files/investigaciones/poblac ion/seriesp85_20/EstimacionesProyecciones1985_02 0.xls

4. García Hernández M, Martínez Sellarés R. Proceso de Envejecer. En: Elsevier, editor. Enfermería y envejecimiento. España: Masson; 2012. p. 10-25.

5. Díaz R, Marulanda F, Martínez MH. Prevalencia de deterioro cognitivo y demencia en mayores de 65 años en una población urbana colombiana. Rev Acta Neurológica Colomb. 2013;29(3):141-51.

6. Custodio N, Herrera E, Lira D, Montesinos R, Linares J, Bendezú L. Deterioro cognitivo leve : ¿dónde termina el 
envejecimiento normal y empieza la demencia? An la Fac Med. 2012;73(4):321-30.

7. Lobo A, Saz P, Marcos G. MMSE: Examen Cognoscitivo Mini-Mental. Madrid: TEA Ediciones; 2002.

8. Ministerio de Salud República de Colombia. Resolución número 8430 de 1993. Bogotá: Ministerio de Salud; $1993 . \quad$ Disponible en: https://www.minsalud.gov.co/sites/rid/Lists/Bibliote caDigital/RIDE/DE/DIJ/RESOLUCION-8430-DE1993.PDF

9. Tribunal Nacional Ético de Enfermería. Ley 911 de 2004. Bogotá: Tribunal Nacional Ético de Enfermería; 2004 .

10. Congreso de Colombia. Ley No 161621 Ene 2013. Bogotá: Congreso de Colombia; 2013. Disponible en: http://wsp.presidencia.gov.co/Normativa/Leyes/Docu ments/2013/LEY 1616 DEL 21 DE ENERO DE 2013.pdf

11. Leite MT, Castioni D, Kirchner RM, Hildebrandt LM. Capacidad funcional y nivel cognitivo de residentes en una comunidad en el sur de Brasil adultos mayores Capacidade funcional e nível cognitivo de idosos residentes em uma comunidade do sul do Brasil Functional capacity and cognitive level of elderly resi. Enferm Glob. 2015;(37):1-11.

12. Bulech GM, Butcher HK, Dochterman JM, Wagner CM. Clasificación de intervenciones de enfermería (NIC). Sexta edic. Barcelona: Elsevier; 2014.634 p.

13. Rodriguez M, Sánchez J. Reserva cognitiva y demencia. An Psicol. 2004;20(2):175-86.

14. Abarca J, Chino B, Llacho M, Gonzalez K, Mucho K, Vázques $\mathrm{R}$, et al. Relación entre Educación, Envejecimiento y Deterioro Cognitivo en una Muestra de adultos mayores de arequipa. Rev Neuropsicol Neuropsquiatria y Neurociências. 2008;8(2):1-9.

15. Durán-Badillo T, Aguilar R, Martínez M, Rodriguez T, Gutierrez G VL. Depresión y función cognitiva de adultos mayores de una comunidad urbano marginal. Enfermería Univ. 2013;10(2):36-42.

16. Mías C, Sassi M, Masih ME, Querejeta A KR. Deterioro cognitivo leve: estudio de prevalencia y factores sociodemográficos en la ciudad de Córdoba, Argentina. Rev Neurol. 2007;44(12):733-8.

17. Ávila-Oliva MM. Caracterización de la familia del anciano con deterioro cognitivo. Rev Cuba Salud Pública. 2012;38(2):246-52.

18. Corte Constitucional de la República de Colombia. Constitución Política de Colombia. Bogotá: Corte Constitucional; 2015. p. 121.

19. Pinillos-pati $Y$, Prieto-su E. Funcionalidad física de personas mayores institucionalizadas y no institucionalizadas en Barranquilla, Colombia. 2012;14(3):438-47.

20. Melguizo-Herrera E, Ayala-Medrano S, Grau-Coneo M, Merchán Duitama A, Montes-Hernández T, PayaresMartínez C R-VT. Calidad de vida de adultos mayores en centros de protección social en Cartagena (Colombia). Aquichan. 2014;14(4):537-48.

21. León $\mathrm{R}$, Elena $\mathrm{R}$, Millán $\mathrm{N}$, Arévalo $\mathrm{R}$, Escartín $\mathrm{M}$. Factores de riesgo para deterioro cognitivo y funcional en el adulto mayor. Rev Med Inst Mex Seguro Soc. 2009;47(3):277-84. 